\title{
A Nonconvex Nonsmooth Regularization Method with Structure Tensor Total Variation is
}

\author{
Zhuo-Xu Cui, Qibin Fan*, Yichuan Dong, Tong Liu \\ School of Mathematics and Statistics, Wuhan University, Wuhan 430072, P.R. China
}

\begin{abstract}
In this paper, a novel regularization method for image restoration and reconstruction is introduced which is accomplished by adopting a nonconvex nonsmooth penalty that depends on the eigenvalues of structure tensor of the underlying image. At first, an alternating minimization scheme is developed in which the problem can be decomposed into three subproblems, two of them are convex and the remaining one is smooth. Then, the convergence of the sequence which generate by the alternating minimization algorithm is proved. Finally, the efficient performance of the proposed method is demonstrated through experimental results for both grayscale and vector-value images.

Keywords: Nonconvex nonsmooth regularization, structure tensor, image restoration and reconstruction, alternating minimization methods.
\end{abstract}

\section{Introduction}

Image processing, such as Computerized Tomography, CCD sensors (see [14, 24, 23]) e.t.c., plays an important role in major of engineering and medicine. The problem only provides indirect information of the unknowns, our purpose is to determine the unknowns from the measure by constructing stable maps, while considering the uniqueness and stability of the inverse maps [16]. In this paper, we consider a basic forward image

\footnotetext{
This work is supported by the National Science Foundation of China under Grant 61179039 and the National Key Basic Research Development Program (973 Program) of China under Grant 2011 CB707100.

* Corresponding author

Email addresses: zhuoxucui@whu.edu.cn (Zhuo-Xu Cui), qbfan@whu.edu.cn (Qibin Fan), ycdong@whu.edu.cn (Yichuan Dong), tongliu@whu.edu.cn (Tong Liu)

Preprint submitted to J. Vis. Commun. Image R.

October 29, 2016
} 
restoration model, which can be formulated as

$$
g=A u+n,
$$

where $u=\left[u_{1}(x), \ldots, u_{M}(x)\right]: \Omega \in \mathbb{R}^{2} \mapsto \mathbb{R}^{M}$ is the original vector-value image with $M$ channels, $n=\left[n_{1}(x), \ldots, n_{M}(x)\right]: \mathbb{R}^{2} \mapsto \mathbb{R}^{M}$ accounts for the perturbations and $A$ is a linear operator that represents for a blurring in optics or a transform operator in

5 medical tomography. We would like to restore an image $u$ from the measurement $g$ where $A u$ should be closed to $g$ in the forward image restoration model and $u$ need to satisfy some prior requirements. For most cases of practical interest, the operator $A$ is either ill-conditioned or singular. And Regularization is the popular method to deal with this kind of ill-posedness problems $[8,30]$. The restoration of $u$ is cast as a minimization 10 problem:

$$
\mathcal{J}(u)=\|A u-g\|^{2}+\tau \Phi(u)
$$

Such a cost function consists of two terms, the first is a data fidelity term which measures how well a candidate solution explains the observed data, the second is a regularized term which encodes any avaliable prior information about the underlying image and $\tau$ is a positive regularized parameter that controls the tradeoff between these two terms.

As state earlier, total variation (TV) semi-norm have been applied to grayscale images $(M=1)$ processing $[6,5]$

$$
\Phi(u):=\sup \left\{\int_{\Omega} u \nabla \cdot(v): v \in \mathcal{C}_{0}^{\infty}\left(\Omega ; \mathbb{R}^{n}\right),|v| \leq 1\right\}
$$

${ }_{15}$ where $u$ is defined on the bounded domain $\Omega \subset \mathbb{R}^{n}$ and $\mathcal{C}_{0}^{\infty}\left(\Omega ; \mathbb{R}^{n}\right)$ denote the space of arbitrarily differentiable functions with compact support. TV regularizer is convex and rotation-invariant which preserve corners and edges well. Due to this favorable property, TV have been extended to vector-value images [2]. However, a known drawback of TV is that favors piecewise-constant solutions and, thus it can create strong staircase artifacts in smooth regions of the image. In addition, TV relies only on one of the points of $\Omega$ without taking into account the available information from its neighborhood.

In literature [28, 29], a more sophisticated descriptor of image variations has been proposed: structure tensor TV function. It generalizes several existing variational penalties, including TV and vectorial extension of TV, and has the ability to capture first-order 
25 information around a local neighborhood. As a consequence, the structure tensor TV function can provide more robust measures of image variation. From another point of view, the discrete structure tensor TV function can be formulate as

$$
\Phi(u)=\sum_{i=1}^{N_{1}} \sum_{k=1}^{N_{2}}\left\|\left(\sqrt{\lambda_{i, k}^{+}}, \sqrt{\lambda_{i, k}^{-}}\right)\right\|_{1},
$$

where $\lambda_{i, k}^{+}$and $\lambda_{i, k}^{-}$denote the two nonnegative eigenvalues of the discrete structure tensor at pixel $(i, k)$ (see Sect. 2 for detail).

structure tensor TV function is the summation of the $\left\|\left(\sqrt{\lambda_{i, k}^{+}}, \sqrt{\lambda_{i, k}^{-}}\right)\right\|_{1}$ of the underlying image over the domain. The combination leads to an $\ell_{1}$ weighting that dose not overpenalize high variation of $u$, but the $\ell_{1}$ weighting minimization problem may yield the all the $\left\{\left\|\left(\sqrt{\lambda_{i, k}^{+}}, \sqrt{\lambda_{i, k}^{-}}\right)\right\|_{1}\right\}$ with lower sparse rate than the real one. Another limitation of the $\ell_{1}$-norm minimization is its bias caused by shrinking all the $35\left\{\left\|\left(\sqrt{\lambda_{i, k}^{+}}, \sqrt{\lambda_{i, k}^{-}}\right)\right\|_{1}\right\}$ toward zero simultaneously [15]. In order to overcome the limitations of $\ell_{1}$ weighting minimization problem, many nonconvex penalty functions have been proposed, include $\ell_{p}$-norm $(0<p<1)[12]$, Smoothly Clipped Absolute Deviation (SCAD) [10], Minimax Concave Penalty (MCP) [36], Nonconvex Nonsmooth Potential Functions (PF) [20, 21, 31], and so on. What's more, In [31], we know that nonconvex 40 regularization methods are effective for restoring images with neat edges. The work of Nikolova, Ng and Tam [20] showed that the nonconvex image restoration models enjoy better properties than the convex ones and the nonconvex nonsmooth PF model also performs well. Therefore, we are interested in studying the nonconvex nonsmooth PF penalty with structure tensor TV.

The main contribution of this work: (1) We proposed a nonconvex nonsmooth regularizer which penalizes the image variation at every point of the domain by taking into account the information in a local neighborhood. This is accomplished by adopting a nonconvex nonsmooth penalty that depends on the eigenvalues of structure tensor. (2) Due to the nonconvex nonsmooth property of our proposed regularizer, minimizing the 50 cost function (1.1) become challengeable. Thus, we propose an alternating minimization scheme in which the problem can be decomposed into three subproblems, the two of them are convex and the remaining one is smooth. Therefore, some powerful optimization tools can be applied to deal with these three subproblems. (3) We prove that our 
alternating minimization algorithm converges to a critical point.

The rest of this paper is organized as follows. In Section 2, we focus on the nonconvex nonsmooth regularization model and present an alternating minimization algorithm. In Section 3, we study the convergence of our proposed algorithm. In Section 4, we assess the reconstruction and restoration performance of our algorithm by designing some numerical examples. Conclusions are drawn in section 5, while some mathematical proofs are ${ }_{60}$ provided in Appendix.

Notion: To simplify the presentation, we use $\|\cdot\|$ to denote $L_{2}$-norm in continuous case and to denote $\ell_{2}$-norm in discrete case. For any $x \in \mathbb{R}^{d}$ and $p \geq 1,\|\cdot\|_{p}$ denote the standard $\ell_{p}$-norm in $\mathbb{R}^{d}$, i.e., $\|x\|_{p}=\left(\sum_{i=1}^{d}\left|x_{i}\right|^{p}\right)^{\frac{1}{p}}$. For any $Y \in \mathbb{R}^{n_{1} \times n_{2}}$ We denote $\|\cdot\|_{*}$ as nuclear-norm of $Y$, i.e., $\|Y\|_{*}=\sum_{i=1}^{n} \sigma_{i}(Y)$, where $\sigma_{i}$ is the $i$ largest singular value of $Y$ and $n$ is the number of singular values, and $\|\cdot\|_{F}$ as Frobenius norm of $Y$, i.e, $\|Y\|_{F}=\left(\sum_{i=1}^{n} \sigma_{i}^{2}(Y)\right)^{\frac{1}{2}}$. We use $\delta$ to denote the Dirac distribution, i.e, $\delta(0)=\infty$, $\delta(t)=0, \forall t \neq 0$ and $\int_{-\infty}^{+\infty} \delta(t) d t=1$. We say that $u \in \mathcal{C}^{l}(\Omega)$ if it is $l$ times continuously differentiable functions with compact support, particularly, $\mathcal{C}_{0}^{\infty}\left(\Omega ; \mathbb{R}^{n}\right)$ denote the space of infinitely differentiable functions with compact support. Let $\mathcal{X}, \mathcal{Y}$ be Hilbert spaces and $\mathcal{G} \subset \mathcal{X}$ be a dense linear subspace and $A: \mathcal{G} \rightarrow \mathcal{Y}$ a linear operator. There exists a unique linear operator $A^{*}: \operatorname{Dom}\left(A^{*}\right) \subset \mathcal{Y} \rightarrow \mathcal{X}$ such that $\left\langle A^{*} y, x\right\rangle=\langle y, A x\rangle$, for any $x \in$ $\mathcal{G}$ and $y \in \operatorname{Dom}\left(A^{*}\right)$, where $\operatorname{Dom}\left(A^{*}\right):=\{y \in \mathcal{Y}: x \rightarrow\langle y, A x\rangle$ is continuous on $\mathcal{G}\}$. For any convex function $h$ and $x, y \in \mathbb{R}^{d}, \nabla h(x)(\partial h(x))$ is the set of gradient (subgradient) at $x$, in particular,

$$
\partial h(x)=\left\{\xi \in \mathbb{R}^{d}: h(y) \geq h(x)+\langle\xi, y-x\rangle\right\}
$$

For any function $g: \mathbb{R}^{n_{1} \times n_{2}} \rightarrow \mathbb{R}$ and $Y, \Delta Y \in \mathbb{R}^{n_{1} \times n_{2}}$, operator $D g(Y): \mathbb{R}^{n_{1} \times n_{2}} \rightarrow \mathbb{R}$ denote the Gateaux derivatives at $Y$, in particular,

$$
\lim _{t \rightarrow 0} \frac{1}{t}|g(Y+t \Delta Y)-g(Y)-t\langle D g(Y), \Delta Y\rangle|=0 .
$$

For a tensor $Z \in \mathbb{R}^{n_{1} \times n_{2} \times n_{3}}$, we use $Z(i,:,:)$ to denote the matrix $Z(i, j, k) \in \mathbb{R}^{n_{2} \times n_{3}}$ with fixed $i$. One important property which we will use later on is the Kurdyka-Lojasiewicz (KL) property.

Definition 1.1. (KL function). A proper function $f: \mathbb{R}^{p} \rightarrow \mathbb{R}$ is said to have the Kurdyka-Eojasiewicz (KL) property at $\widehat{x} \in \operatorname{Dom}(\partial f)\left(\equiv\left\{z \in \mathbb{R}^{p}: \partial f(z) \neq \varnothing\right\}\right)$ if there 
exist $\eta \in[0, \infty)$, a neighborhood $\mathcal{V}$ of $\widehat{x}$, and a continuous concave function $\theta:[0, \eta) \rightarrow \mathbb{R}_{+}$ such that

- $\theta(0)=0$;

- $\theta$ is continuous differentiable on $(0, \eta)$;

- for all $z \in(0, \eta), \theta^{\prime}(z)>0$;

- for all $z \in \mathcal{V} \bigcap[f(\widehat{z})<f<f(\widehat{z})+\eta]$, the Kurdyka-Eojasiewicz inequality and

$$
\theta^{\prime}(f(z)-f(\widehat{z})) \operatorname{dist}(0, \partial f(z)) \geq 1
$$

hold, where $\operatorname{dist}(z, \mathcal{U})=\inf \|y-z\|, y \in \mathcal{U}$, for any $\mathcal{U} \subset \mathbb{R}^{p}$. Moreover, proper semicontinuous functions which satisfy the Kurdyka-Eojasiewicz inequality at each point of $\operatorname{Dom}(\partial f)$ are called KL functions.

\section{Nonconvex Structure Tensor TV}

\section{2.1. The Structure Tensor and Problem Formulation}

In this subsection, we begin with reviewing the theory behind the structure tensor $[18,19,9]$ and then propose our nonconvex nonsmooth regularization problem. First, we assume that vector-valued image $u \in W^{1,2}\left(\mathbb{R}^{2}, \mathbb{R}^{M}\right)$, i.e. Sobolev space, hence $u=$ $\left(u_{1}, \ldots, u_{M}\right), u_{h}=\left(\frac{\partial u_{1}}{\partial x^{h}}, \ldots, \frac{\partial u_{M}}{\partial x^{h}}\right), h=1,2$ is a basis for 2 -dimensional vector space of tangent vector of $W^{1,2}\left(\mathbb{R}^{2}, \mathbb{R}^{M}\right)$ at $u=u(x)$ [35]. The variation of the vector-valued image $u$ at any specific image point $x$ is given by

$$
\mathrm{d} u^{2}=\left[\mathrm{d} x^{1}, \mathrm{~d} x^{2}\right] S u(x)\left[\mathrm{d} x^{1}, \mathrm{~d} x^{2}\right]^{T}
$$

where $S u$ and $J u$ are defined as

$$
\begin{gathered}
S u(x)=\left[J u^{T} J u\right](x), \\
J u(x)=\left[\nabla u_{1}(x), \ldots, \nabla u_{M}(x)\right]^{T},
\end{gathered}
$$

[d $\left.x^{1}, \mathrm{~d} x^{2}\right]$ is the dual basis of $u_{h}$ and $S u$ is the so called structure tensor [11]. In order to use the information from the neighborhood, nonlocal structure tensor has been proposed

$$
S_{K} u(x)=K *\left[J u^{T} J u\right](x),
$$


where $*$ denotes the convolution operation, $K(x)$ is a nonnegative, rotationally symmetric convolution kernel.

In image applications, we are interested in finding the direction through point $x$ along $u$ which has the maximum and minimum rate of change. Let $\nu$ be an arbitrary unit two-dimensional direction vector, the nonlocal directional variation is given by

$$
R M S_{K}\left(\|\partial u / \partial \nu\|_{2}\right)=\sqrt{K *\|\partial u / \partial \nu\|_{2}^{2}}=\sqrt{\nu^{T}(S u) \nu}
$$

Let $\lambda_{+}=\lambda_{+}\left(S_{K} u(x)\right), \lambda_{-}=\lambda_{-}\left(S_{K} u(x)\right)$ to be the ordered eigenvalues of $S_{K} u(x)$, namely, $\lambda_{+} \geq \lambda_{-}$, and $\theta_{+}, \theta_{-}$to be the corresponding unit eigenvectors. Therefore, (2.2) can be reformulated as

$$
R M S_{K}\left(\|\partial u / \partial \nu\|_{2}\right)=\sqrt{\nu^{T}\left[\theta_{+}, \theta_{-}\right] \Lambda\left[\theta_{+}, \theta_{-}\right]^{T} \nu}
$$

where $\Lambda=\operatorname{diag}\left(\lambda_{+}, \lambda_{-}\right)$. If we choose $\nu=\theta_{+}$, then the maximum of $R M S_{K}$ is equal to $\sqrt{\lambda_{+}}$, and if we choose $\nu=\theta_{-}$, then the minimum of $R M S_{K}$ is $\sqrt{\lambda_{-}}$. It's concluded

85 that the structure tensor could measure the geometry of image structures in each point, eigenvalues $\theta_{+}$and $\theta_{-}$describe the orientation of maximum and minimum directional variation of $u$ and $\sqrt{\lambda_{+}}$and $\sqrt{\lambda_{-}}$describe measures of these variations.

In this paper, we introduce a novel a nonconvex nonsmooth regularized method which base on the nonlocal structure tensor. And the new regularizer $\Phi(u)$ reads:

$$
\Phi(u):=\int_{\Omega}\left[\phi\left(\sqrt{\lambda_{+}}\right)+\phi\left(\sqrt{\lambda_{-}}\right)\right] \mathrm{d} x
$$

where $\phi: \mathbb{R} \mapsto \mathbb{R}^{+}$is a potential function (PF). Particularly, when we choose $K=\delta$ (Dirac function), and consider the grayscale image $(M=1)$, then $(2.3)$ could be expressed as follows:

$$
\Phi(u)=\int_{\Omega} \phi\left(\|\nabla u\|_{2}\right) \mathrm{d} x .
$$

And if we choose PF as $\phi(t)=|t|$, then, the regularizer $\Phi(u)$ is equal to TV semi-norm. With the result that our proposed method could generalize TV. In this work, we are interested in choosing a nonconvex nonsmooth PF. Due to the nonconvex nonsmooth property of our proposed regularizer $\phi$, minimizing the cost function (1.1) become challengeable. From [7], we known that PF can be decomposed into $\phi(\cdot)=\psi(\cdot)+\phi^{\prime}\left(0^{+}\right)|\cdot|$ and $\psi \in \mathcal{C}^{2}$. Subsequently, we can decompose (2.3) into two term:

$$
\Phi(u)=\Psi(u)+\alpha \int_{\Omega}\left\|J_{K} u\right\|_{*} \mathrm{~d} x,
$$


where

$$
\Psi(u):=\int_{\Omega}\left[\psi\left(\sqrt{\lambda_{+}}\right)+\psi\left(\sqrt{\lambda_{-}}\right)\right] \mathrm{d} x
$$

90 $\alpha=\phi^{\prime}\left(0^{+}\right)$and $\|\cdot\|_{*}$ denote the nuclear norm. $\int_{\Omega}\left\|J_{K} u\right\|_{*} \mathrm{~d} x$ is convex and $\Psi(u)$ is Gateaux differentiable which will be proved in the next subsection. Then, problem (1.1) can be reformulated as

$$
\mathcal{J}(u)=\|A u-g\|^{2}+\tau \Psi(u)+\tau \cdot \alpha \int_{\Omega}\left\|J_{K} u\right\|_{*} \mathrm{~d} x,
$$

In the next subsection, we would use an alternating algorithm to solve problem (2.4).

\subsection{Discrete Nonconvex Structure Tensor TV and Algorithm}

In this subsection, we transform the above problem (2.4) into discrete form and design alternating minimization algorithm via introducing a auxiliary variable. We assume that the discrete vector-value image $u=\left[u_{1}, \ldots, u_{M}\right]$, and $u_{j} \in \mathbb{R}^{N_{1} \times N_{2}}(j=1, \ldots, M)$ is defined on a rectangular grid with size $N_{1} \times N_{2}$. We assume in (2.1), $K$ is a nonnegative, rotation-invariable and discretized convolution kernel with size $\left(2 L_{K}+1\right) \times\left(2 L_{K}+1\right)$ and $i=1, \ldots, N_{1}, k=1, \ldots, N_{2}$. The patch-based Jacobian is defined in [18], $J_{K}$ : $\mathbb{R}^{N_{1} \times N_{2} \times M} \mapsto \mathbb{R}^{N_{1} \times N_{2} \times L_{K} \cdot M \times 2}$ and $L=\left(2 L_{k}+1\right)^{2}$. The patch-based Jacobian reads:

$$
\left[J_{K} u\right](i, k)=[\tilde{\nabla} u(i, k, 1), \ldots, \tilde{\nabla} u(i, k, M)]^{T}
$$

where

$$
\begin{gathered}
{[\tilde{\nabla} u]=\left[T_{-L_{K},-L_{K}} \circ \nabla u, \ldots, T_{L_{K},-L_{K}} \circ \nabla u, \ldots, T_{L_{K}, L_{K}} \circ \nabla u\right],} \\
T_{x^{\prime}, y^{\prime}} \circ \nabla u=\sqrt{K\left(x^{\prime}, y^{\prime}\right)} \cdot \nabla u\left(i-x^{\prime}, k-y^{\prime}, j\right), x^{\prime}, y^{\prime}=-L_{K} \ldots, L_{K} .
\end{gathered}
$$

We have

$$
\left\|J_{K} u\right\|_{*}=\left\|\left(\sqrt{\lambda_{+}}, \sqrt{\lambda_{-}}\right)\right\|_{1},
$$

where $\lambda_{+}$and $\lambda_{-}$are the eigenvalues of $S_{K} u(x)$. Then, the discrete form of $\int_{\Omega}\left\|J_{K} u\right\|_{*} d \Omega$ in (2.4) is given by (1.2) and the discrete form of $\Psi(u)$ in (2.4) can be formulated as

$$
\tilde{\Psi}(u)=\sum_{i=1}^{N_{1}} \sum_{k=1}^{N_{2}}\left[\psi\left(\sqrt{\lambda_{i, k}^{+}}\right)+\psi\left(\sqrt{\lambda_{i, k}^{-}}\right)\right]
$$


In order to develop an alternating scheme which have been extended into many fields [32, $33,34]$, we introduce an auxiliary variable $W \in \mathbb{R}^{N_{1} \times N_{2} \times L_{K} \cdot M \times 2}$. Then, the augmented Lagrangian function of discrete form of (2.4) can be reformulated as

$$
\begin{aligned}
L_{\rho}(u, W, P)= & \sum_{i=1}^{N_{1}} \sum_{k=1}^{N_{2}}\left(\tau \cdot \alpha\left\|W_{j, k}\right\|_{*}+\frac{\rho}{2}\left\|\left(J_{K} u\right)_{i, k}-W_{i, k}\right\|_{F}^{2}-\left\langle P_{i, k},\left(J_{K} u\right)_{i, k}-W_{i, k}\right\rangle\right) \\
& +\frac{1}{2} \sum_{j=1}^{M}\left\|A u_{j}-g_{j}\right\|_{F}^{2}+\tau \tilde{\Psi}(u) .
\end{aligned}
$$

where $P \in \mathbb{R}^{N_{1} \times N_{2} \times L_{K} \cdot M \times 2}$ is the Lagrangian multiplier, $W_{i, k}=W(i, k,:,:), P_{i, k}=$ $P(i, k,:,:),\left(J_{K} u\right)_{i, k}=\left(J_{K} u\right)(i, k,:,:)$, and $\rho>0$ is the penalty parameter for the violation of the linear constraint. To simplify the presentation, we use $\|A u-g\|_{F, 1}^{2},\|W\|_{*, 1}$, $\left\|J_{K} u-W\right\|_{F, 1}^{2}$ and $\left\langle P, J_{K} u-W\right\rangle$ to represent $\sum_{j=1}^{M}\left\|A u_{j}-g_{j}\right\|_{F}^{2}, \sum_{i=1}^{N_{1}} \sum_{k=1}^{N_{2}}\left\|W_{j, k}\right\|_{*}$, $\sum_{i=1}^{N_{1}} \sum_{k=1}^{N_{2}}\left\|\left(J_{K} u\right)_{i, k}-W_{i, k}\right\|_{F}^{2}$ and $\sum_{i=1}^{N_{1}} \sum_{k=1}^{N_{2}}\left\langle P_{i, k},\left(J_{K} u\right)_{i, k}-W_{i, k}\right\rangle$, respectively. Subsequently, the Lagrangian function can be reformulate as

$$
L_{\rho}(u, W, P)=\frac{1}{2}\|A u-g\|_{F, 1}^{2}+\tau \tilde{\Psi}(u)+\tau \cdot \alpha\|W\|_{*, 1}+\frac{\rho}{2}\left\|J_{K} u-W\right\|_{F, 1}^{2}-\left\langle P, J_{K} u-W\right\rangle
$$

Given $\left(u^{(s-1)}, W^{(s-1)}, P^{(s-1)}\right)$, the iteration scheme of problem (2.5) can be described as follows:

$$
\left\{\begin{array}{l}
W^{(s)} \in \arg \min _{W \in \mathbb{R}^{N_{1} \times N_{2} \times L_{K} \cdot M \times 2}} L_{\rho}\left(W, u^{(s-1)}, P^{(s-1)}\right) ; \\
u^{(s)} \in \arg \min _{u \in \mathbb{R}^{N_{1} \times N_{2}}} L_{\rho}\left(W^{(s)}, u, P^{(s-1)}\right) \\
P^{(s)}=P^{(s-1)}-\rho\left(J_{K} u^{(s)}-W^{(s)}\right) .
\end{array}\right.
$$

where arg min denotes the minimal set to an optimization problem.

Similar to $[27,26,25], W$-subproblem can be formulated as matrix factorization problem:

$$
\begin{aligned}
W^{(s)} & \in \arg \min _{W \in \mathbb{R}^{N_{1} \times N_{2} \times L_{K} \cdot M \times 2}} \frac{\rho}{2}\left\|W-J_{K} u^{(s-1)}\right\|_{F, 1}^{2}+\alpha \tau\|W\|_{*, 1}-\left\langle P^{(s-1)}, W-J_{K} u^{(s-1)}\right\rangle \\
& =\arg \min _{W \in \mathbb{R}^{N_{1} \times N_{2} \times L_{K} \cdot M \times 2}} \frac{1}{2}\left\|\left(J_{K} u^{(s-1)}+\frac{1}{\rho} P^{(s-1)}\right)-W\right\|_{F, 1}^{2}+\alpha \tau\|W\|_{*, 1},
\end{aligned}
$$

where $\iota=\frac{\tau \alpha}{\rho}$. According to [3], it's easy to show the solution of (2.6) as

$$
W_{i, k}^{(s)}=\mathcal{D}_{\iota}\left(\left(Y^{(s-1)}\right)_{i, k}\right), i=1, \ldots, N_{1}, k=1, \ldots, N_{2},
$$


where $Y^{(s)}=J_{K} u^{(s-1)}+\frac{1}{\rho} P^{(s-1)}, \mathcal{D}_{\iota}$ is the soft-thresholding operator, $\mathcal{D}_{\iota}=U \mathcal{D}_{\iota}(\Sigma) V^{*}$, $\mathcal{D}_{\iota}(\Sigma)=\operatorname{diag}\left(\left\{\sigma_{i}-\kappa\right\}_{+}\right), t_{+}$is the positive part of $t$, namely, $t_{+}=\max (0, t)$ and $\left(J_{K} u^{(s-1)}\right)_{i, k}=U \Sigma V^{*}$ is the singular value decomposition (SVD) of matrix $\left(Y^{(s)}\right)_{i, k}$.

The $u$-subproblem can be formulated as follows

$u^{(s)}=\arg \min _{u \in \mathbb{R}^{N_{1} \times N_{2} \times M}} \frac{1}{2}\|A u-g\|_{F, 1}^{2}+\tau \tilde{\Psi}(u)+\frac{\rho}{2}\left\|J_{k} u-W^{(s)}\right\|_{F, 1}^{2}-\left\langle P^{(s-1)}, W^{(s)}-J_{K} u\right\rangle$,

we could use Quasi-Newton's method to solve this optimization problem (2.7):

$$
\begin{gathered}
\left(A^{*} A+\rho J_{K}^{*} J_{K}\right) \Delta u=A^{*}\left(g-A u^{(s-1)}\right)-\tau D_{u} \tilde{\Psi}\left(u^{(s-1)}\right)+\rho\left(W^{(s)}-J_{K} u^{(s-1)}\right)-J_{K}^{*} P^{(s-1)}, \\
u^{(s)}=u^{(s-1)}+\Delta u,
\end{gathered}
$$

where $A^{*}$ is the adjoint of $A$, and $J_{K}^{*}$ is the adjoint of $J_{K}$. In order to get $\Delta u$, in practice, it may be expensive to solve the above linear system directly, we can apply the conjugate method [17] for solving it. For the sake of simplicity, let $C=A^{*} A+\rho J_{K}^{*} J_{K}$, and $E_{s-1}=A^{*}\left(g-A u^{(s-1)}\right)-\tau D_{u} \tilde{\Psi}\left(u^{(s-1)}\right)+\rho\left(W^{(s)}-J_{K} u^{(s-1)}\right)-J_{K}^{*} P^{(s-1)}$. Let $\Delta u_{0}=0, \hat{R}_{0}=C \Delta u_{0}-E_{s-1}$ and $\hat{P}_{0}=-\hat{R}_{0}$, and then the sequence $\left\{\Delta u_{i}\right\}$ can be computed iteratively as

$$
\left\{\begin{array}{l}
\alpha_{i}=-\frac{\left\langle\hat{R}_{i}, \hat{P}_{i}\right\rangle}{\left\langle\hat{P}_{i}, C \hat{P}_{i}\right\rangle}, \\
\Delta u_{i+1}=\Delta u_{i}+\alpha_{i} \hat{P}_{i}, \\
\hat{R}_{i+1}=C \Delta u_{i+1}-E_{s-1}, \\
\beta_{i+1}=\frac{\left\langle\hat{R}_{i+1}, C \hat{P}_{i}\right\rangle}{\left\langle\hat{P}_{i}, C \hat{P}_{i}\right\rangle}, \\
\hat{P}_{i+1}=-\hat{R}_{i+1}+\beta_{i+1} \hat{P}_{i}
\end{array}\right.
$$

Next, we will compute the Gateaux derivatives of $\tilde{\Psi}(u)$. It has been show in $[20]$ that $\psi \in \mathcal{C}^{2}$ on $(0, \infty)$. Thus, we have the following:

Proposition 2.1. The Gateaux derivatives of $\tilde{\Psi}(u)$ is

$$
\left(D_{u} \tilde{\Psi}(u)\right)_{i, k}=2 J_{K}^{*} P_{i, k}^{*}\left(\Theta_{i, k} P_{i, k} J_{K} u\right),
$$

110 where $\left(D_{u} \tilde{\Psi}(u)\right)_{i, k}=\left(D_{u} \tilde{\Psi}(u)\right)(i, k,:), \Theta_{i, k}=\frac{\partial \psi\left(\sqrt{\lambda_{i, k}^{+}}\right)}{\partial \lambda_{i, k}^{+}} \cdot \theta_{i, k}^{+}\left(\theta_{i, k}^{+}\right)^{T}+\frac{\partial \psi\left(\sqrt{\lambda_{i, k}^{-}}\right)}{\partial \lambda_{i, k}^{-}} \cdot \theta_{i, k}^{-}\left(\theta_{i, k}^{-}\right)^{T}$, $P_{i, k}: \mathbb{R}^{N_{1} \times N_{2} \times L_{K} \cdot M \times 2} \rightarrow \mathbb{R}^{L_{K} \cdot M \times 2}, P_{i, k} J_{K} u=\left(J_{K} u\right)_{i, k},\left(J_{K} u\right)_{i, k}=\left(J_{K} u\right)(i, k,:,:)$, and $P_{i, k}^{*}$ is the adjoint of $P_{i, k}$. 
The proof of Proposition 2.1 is given in Appendix (A). Based on the analysis above, we give a basic framework of the alternating minimization scheme for solving our nonconvex nonsmooth augmented model as follows:

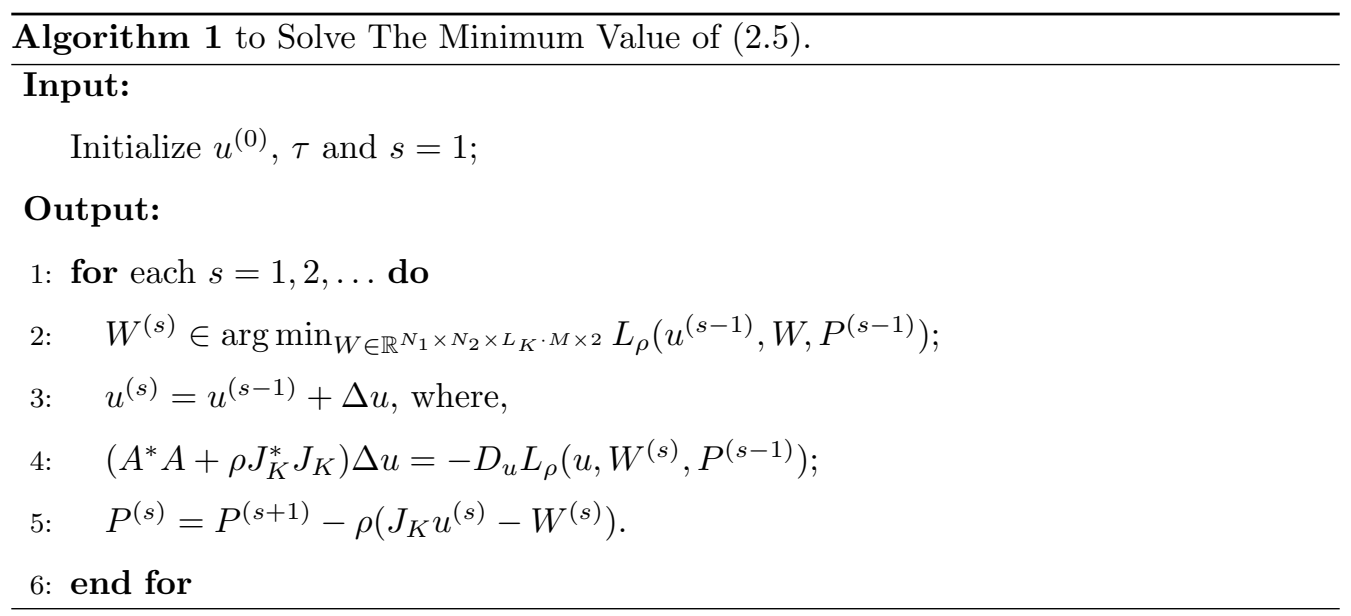

\section{Convergence Analysis}

The main aim of this section is to show the that Algorithm 1 converges to a critical point of (2.5). Now we can establish the properties of $\left(u^{(s)}, W^{(s)}, P^{(s)}\right)$, and use them to 120 show the convergence of Algorithm 1.

Proposition 3.1. Suppose that $\underline{\lambda}-\frac{L}{2}>0$, where $\underline{\lambda}=\lambda_{\min }\left(A^{*} A+\rho J_{K}^{*} J_{K}\right)$ and $L$ is the Lipschitz constant of $D_{u} L_{\rho}\left(u, W^{(s)}, P^{(s-1)}\right)$. Then, the sequences $\left(u^{(s)}, W^{(s)}, P^{(s)}\right)$ generated by Algorithm 1, satisfy the following inequalities and equality:

(i)

$$
\begin{aligned}
& \frac{\rho}{2}\left\|J_{K} u^{(s-1)}-W^{(s)}\right\|_{F, 1}^{2}+\tau \alpha\left\|W^{(s)}\right\|_{*, 1}-\left\langle P^{(s-1)}, W^{(s)}-J_{K} u^{(s-1)}\right\rangle+\mu_{1}\left\|W^{(s-1)}-W^{(s)}\right\|_{F, 1}^{2} \\
& \leq \frac{\rho}{2}\left\|J_{K} u^{(s-1)}-W^{(s-1)}\right\|_{F, 1}^{2}+\tau \alpha\left\|W^{(s-1)}\right\|_{*, 1}-\left\langle P^{(s-1)}, W^{(s-1)}-J_{K} u^{(s-1)}\right\rangle .
\end{aligned}
$$


$\frac{1}{2}\left\|A u^{(s)}-g\right\|_{F, 1}^{2}+\tau \Psi\left(u^{(s)}\right)+\frac{\rho}{2}\left\|J_{K} u^{(s)}-W^{(s)}\right\|_{F, 1}^{2}-\left\langle P^{(s-1)}, W^{(s)}-J_{K} u^{(s)}\right\rangle$

$+\mu_{2}\left\|u^{(s-1)}-u^{(s)}\right\|_{F, 1}^{2}$

$\leq \frac{1}{2}\left\|A u^{(s-1)}-g\right\|_{F, 1}^{2}+\tau \Psi\left(u^{(s-1)}\right)+\frac{\rho}{2}\left\|J_{K} u^{(s-1)}-W^{(s)}\right\|_{F, 1}^{2}-\left\langle P^{(s-1)}, W^{(s)}-J_{K} u^{(s-1)}\right\rangle$.

(iii)

$$
\left\langle P^{(s-1)}-P^{(s)}, J_{K} u^{(s)}-W^{(s)}\right\rangle=\rho\left\|P^{(s-1)}-P^{(s)}\right\|_{F, 1}^{2} .
$$

The proof of Proposition 3.1 is given in Appendix (B).

Theorem 3.1. For any $T>0$, and the sequences $\left(u^{(s)}, W^{(s)}, P^{(s)}\right)$ generated by Algorithm 1, there exist a $\kappa>0$ satisfy the following inequality

$$
\sum_{s=1}^{T} \operatorname{dist}\left(\partial L_{\rho}\left(u^{(s)}, W^{(s)}, Z^{(s)}\right), 0\right)^{2} \leq \frac{1}{\kappa}\left[L_{\rho}\left(u^{(0)}, W^{(0)}, P^{(0)}\right)-L_{\rho}\left(u^{(T)}, W^{(T)}, P^{(T)}\right)\right] .
$$

The proof of theorem 3.1 is given in Appendix (C).

Remark 1. According to literature [13], we can use $\operatorname{dist}\left(\partial L_{\rho}\left(u^{(s)}, W^{(s)}, P^{(s)}\right), 0\right)^{2} \leq \epsilon$ to judge approximate stationarity of $\left(u^{(s)}, W^{(s)}, P^{(s)}\right)$, which contrasts to convex optimization, where one uses $\left|L_{\rho}\left(u^{(s)}, W^{(s)}, P^{(s)}\right)-L_{\rho}\left(u^{*}, W^{*}, P^{*}\right)\right|$ or $\left\|u-u^{*}\right\|_{F, 1}^{2}+\| W-$ $W^{*}\left\|_{F, 1}^{2}+\right\| P-P^{*} \|_{F, 1}^{2}$ as criteria for convergence analysis.

135 Remark 2. From (3.4), it is easy to obtain a $\epsilon$-accurate solution in time $o\left(\frac{1}{\epsilon^{2}}\right)$ for our algorithm.

From the definition of $\Psi$ we know that it is a KL function. Our objective is to show that the sequence which is generated by Algorithm 1 converges to a critical point of (2.5) For this purpose we use the KL property to analyze the convergence of the sequence.

Theorem 3.2. Let $\left(u^{(s)}, W^{(s)}, P^{(s)}\right)$ be a sequence generated by Algorithm 1 with an initial point $\left(u^{(0)}, W^{(0)}, P^{(0)}\right)$. If $L_{\rho}$ has the Kurdyka-Eojasiewicz (KL) property at a critical point $\left(u^{*}, W^{*}, P^{*}\right)$ with

$$
L_{\rho}\left(u^{*}, W^{*}, P^{*}\right) \leq L_{\rho}\left(u^{(s)}, W^{(s)}, P^{(s)}\right) \leq L_{\rho}\left(u^{*}, W^{*}, P^{*}\right)+\eta,
$$


and

$$
\begin{aligned}
C & +2 \sqrt{\frac{L_{\rho}\left(u^{(0)}, W^{(0)}, P^{(0)}\right)-L_{\rho}\left(u^{*}, W^{*}, P^{*}\right)}{a}} \\
& +\frac{b}{a} \theta\left(L_{\rho}\left(u^{(0)}, W^{(0)}, P^{(0)}\right)-L_{\rho}\left(u^{*}, W^{*}, P^{*}\right)\right)<\varrho .
\end{aligned}
$$

140 where $C=\left\|u^{(0)}-u^{*}\right\|_{F, 1}^{2}+\left\|W^{(0)}-W^{*}\right\|_{F, 1}^{2}+\left\|P^{(0)}-P^{*}\right\|_{F, 1}^{2}, a=\min \left\{\mu_{1}, \mu_{2}, \rho\right\}$, $b=\tau L^{\prime}+\rho+\frac{1}{\rho}+1+(1+\rho) \bar{\lambda}, \bar{\lambda}=\lambda_{\max } \sqrt{\left(J_{K}^{*} J_{K}\right)}$ and $D \Psi$ is L'-Lipschitz function, then we have

$$
\sum_{s=1}^{+\infty}\left[\left\|u^{(s)}-u^{(s-1)}\right\|_{F, 1}^{2}+\left\|W^{(s)}-W^{(s-1)}\right\|_{F, 1}^{2}+\left\|P^{(s)}-P^{(s-1)}\right\|_{F, 1}^{2}\right]^{\frac{1}{2}} \leq+\infty,
$$

and

$$
\lim _{s \rightarrow+\infty}\left(u^{(s)}, W^{(s)}, P^{(s)}\right)=\left(u^{*}, W^{*}, P^{*}\right) .
$$

The proof of theorem 3.2 is given in Appendix (D).

\section{Numerical Experiments}

\subsection{Experimental settings}

In this section, we present the experimental result to demonstrate the efficiency of our method. Peak-signal-to-noise ratio (PSNR) is used to measure the quality of the restored images, which are defined as

$$
P S N R=10 \cdot \log \frac{255^{2}}{M S E}[d B]
$$

where MSE is the Mean-Squared-Error per pixel.

To show the effectiveness of our proposed methods we compare them with other related methods on several inverse image processing problems. We consider the problems of image reconstruction from a limited number of Fourier measurement and image restorations (image denosing and image debluring). For the first problem, experiments are conducted on images show in Fig. 1. For the image restoration experiments, we present results on both grayscale images and vector-value images. In all the tests, we initialize the image intensities so that they lie in the rang $[0,1]$.

In our experiments, we compare our proposed method with $T V$ [22], $S T V_{\delta}, S T V_{K}$ [18]. And we denote our proposed methods as $N S T V_{\delta}$ with convolution kernel $\delta$ and $N S T V_{K}$ with convolution kernel $K$. We choose $K$ as a Gaussian kernel with standard 
deviation $\sigma=0.5$ pixels, truncated in a $5 \times 5$ pixel window, and choose the potential function $(\mathrm{PF})$ as

$$
\phi(t)=\frac{\alpha|t|}{1+\alpha|t|}, \psi(t)=-\frac{\alpha^{2} t^{2}}{1+\alpha|t|} .
$$

In general, we choose $\alpha=0.5$.

\subsection{Reconstruction from Sparse Fourier Measurement}

In this subsection, we consider the problem of image reconstruction from a limited number of Fourier measurements, i.e., The signals have relatively few nonzero terms or whose coefficients in Fourier basis have relatively few nonzero entries. In this setting, the operator of (1.1) corresponds to $A=M F$, where $F$ denotes the discrete Fourier transform and $M$ is a masking operator that retains only a subset of the available Fourier coefficients [18], and the discrete Fourier transform is given by

$$
(F f)(j, k):=\frac{1}{\sqrt{N_{1} N_{2}}} \sum_{t \in \mathbb{Z}_{N_{1}}, z \in \mathbb{Z}_{N_{2}}} f(t, z) e^{-i 2 \pi\left(\frac{k t}{N_{1}}+\frac{j z}{N_{2}}\right)}
$$

where $f \in \mathbb{R}^{N_{1} \times N_{2}}$, and $N_{1}, N_{2}$ represent the discrete grayscale images matrix dimension, the frequency index $k, z$ ranges over the sets $\mathbb{Z}_{N_{1}}:=\left\{0,1, \ldots, N_{1}-1\right\}, \mathbb{Z}_{N_{2}}:=$ $\left\{0,1, \ldots, N_{2}-1\right\}$.

In literature [4], the strategy based on TV model has been proposed

$$
\min _{f \in \mathbb{R}^{n_{1} \times n_{2}}} \frac{1}{2}\|A f-g\|_{2}^{2}+\tau\|f\|_{T V}
$$

where $\|f\|_{T V}$ is the discrete total variation semi-norm for discrete $f\left(t_{1}, t_{2}\right)$,

$$
\|f\|_{T V}=\sum_{t_{1}, t_{2}} \sqrt{\left|D_{1} f\left(t_{1}, t_{2}\right)\right|^{2}+\left|D_{2} f\left(t_{1}, t_{2}\right)\right|^{2}}
$$

where $D_{1}$ is the finite difference $D_{1} f\left(t_{1}, t_{2}\right)=f\left(t_{1}, t_{2}\right)-f\left(t_{1}-1, t_{2}\right)$ and $D_{2}$ is the finite

difference $D_{2} f\left(t_{1}, t_{2}\right)=f\left(t_{1}, t_{2}\right)-f\left(t_{1}, t_{2}-1\right), 0 \leq t_{1} \leq N_{1}, 0 \leq t_{2} \leq N_{2}$.

The reported experiments are conducted on images shows in Fig. 1. The recovery is performed via the algorithms in this experiment. And we use

$$
\sum_{j=1}^{M}\left\|f_{j}^{(s)}-f_{j}^{(s-1)}\right\|_{F} / \sum_{j=1}^{M}\left\|f_{j}^{(s-1)}\right\|_{F}<10^{-4}
$$

and the maximum iteration step maxit $=500$ as stopping criterion. 


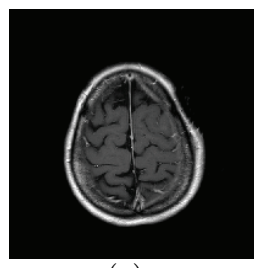

(a)

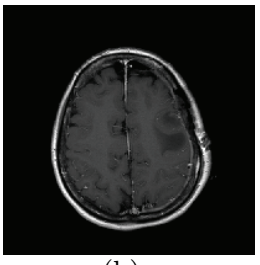

(b)

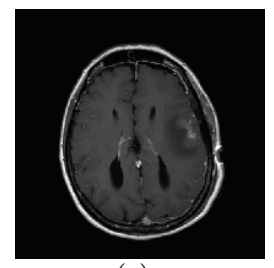

(c)

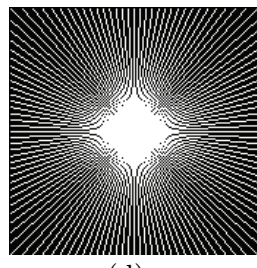

(d)

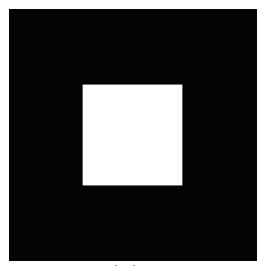

(e)

Figure 1: (a)-(c) Images with size $205 \times 205$; (d) Radial sampling mask with 64 lines; (e) low-frequency sampling with $40 \%$ portion.

Table 1: PSNR comparisons on Fourier image reconstruction for several sampling patterned and noise conditions $\left(N S T V_{\delta}\right.$ represent our proposed nonconvex regularization method).

\begin{tabular}{|c|c|c|c|c|c|c|c|c|c|c|c|c|c|c|c|c|c|c|c|c|c|c|c|c|c|}
\hline \multirow{2}{*}{\multicolumn{2}{|c|}{$\begin{array}{l}\text { Sampling } \\
\text { PSNR }\end{array}$}} & \multirow{2}{*}{\multicolumn{4}{|c|}{$\begin{array}{l}\begin{array}{l}\text { Raddial-32 lines } \\
\text { 20dB }\end{array} \\
\text { 30dB }\end{array}$}} & \multirow{2}{*}{\multicolumn{4}{|c|}{ 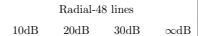 }} & \multicolumn{4}{|c|}{ Radial-64 lines } & \multicolumn{4}{|c|}{ Low-frequency portion 20} & \multicolumn{4}{|c|}{$\begin{array}{l}\text { Low-frequency portion } 25 \\
\text { S }\end{array}$} & \multicolumn{4}{|c|}{ Low-frequency portion 30} \\
\hline & & & & & & & & & & 10dB & $20 \mathrm{~dB}$ & & $\infty \mathrm{dB}$ & $10 \mathrm{~dB}$ & $20 \mathrm{~dB}$ & $30 \mathrm{~dB}$ & $\infty \mathrm{dB}$ & $10 \mathrm{~dB}$ & $20 \mathrm{~dB}$ & $30 \mathrm{~dB}$ & $\infty \mathrm{dB}$ & $10 \mathrm{~dB}$ & $20 \mathrm{~dB}$ & 30dB & odB \\
\hline Brain1 & NSTV 6 & 24.798 & 24.780 & 24.734 & 24.736 & 27.868 & 27.745 & 27.806 & 27.812 & 30.449 & 30.735 & 30.761 & 30.778 & 25.252 & 25.309 & 25.310 & 25.308 & 27.614 & 27.784 & 27.811 & 27.815 & 29.079 & 29.386 & 29.421 & 29.426 \\
\hline & ${ }_{T V}^{T V}$ & 23.956 & 23.907 & 23.862 & 23.866 & 26.865 & 20.568 & 26.499 & 26.506 & 30.239 & 29.614 & 29.581 & 29.663 & 24.988 & 25.035 & 25.031 & 25.031 & 27.459 & 27.631 & 27.659 & 27.668 & 29.107 & 29.551 & 29.608 & 29.631 \\
\hline Brain2 & NSTV $_{6}$ & 24.362 & 24.256 & 24.213 & 24.215 & 27.351 & 27.021 & 27.063 & 26.960 & 29.614 & 29.570 & 29.559 & 29.566 & 23.408 & 23.438 & 23.447 & 23.449 & 24.992 & 25.050 & 25.066 & 25.052 & 26.701 & 26.813 & 26.826 & 26.825 \\
\hline & $T V$ & 23.538 & 23.405 & 23.40 & 23.356 & 26.262 & 25.755 & 25.796 & 25.800 & 29.112 & 28.338 & 28.256 & 28.271 & 23.247 & 23.302 & 23.310 & 23.313 & 24.787 & 24.825 & 24.858 & 24.558 & 26.550 & 26.612 & 26.641 & 26.641 \\
\hline Brain3 & $N_{N T T V_{s}}$ & 23.840 & 23.813 & 23.835 & 23.838 & 26.966 & 26.748 & 26,702 & 26.708 & 29.304 & 29.205 & 29.260 & 29.265 & 23.387 & 23.405 & 23.4411 & 23.407 & 24.907 & 24.991 & 24.998 & 25.000 & 26.147 & 26.299 & 26.321 & 26.321 \\
\hline & ${ }_{T V}^{T V}$ & 23.176 & 23.111 & 23.058 & 23.062 & \begin{tabular}{|l|l|}
26.990 \\
\end{tabular} & 25.519 & 25.580 & 25.585 & 28.870 & 288.065 & 27.980 & 27.985 | & 23.287 & 23.303 & 23.309 & 23.308 & 24.772 & 24.864 & 24.994 & 24.995 & 26.063 & 26.229 & 26.267 & 26.269 \\
\hline
\end{tabular}

To create the measured data we use two different Fourier sampling patterns, namely, a radial mask with 32,48 and 64 radial lines and a low-frequency sampling with $20 \%$, $25 \%$ and $30 \%$ portion. As an additional degradation factor we consider the presence of complex Gaussian noise in Fourier domain of four different levels. These correspond to a signal-to-noise-ratio (SNR) of $\{10,20,30, \infty\} \mathrm{dB}$, and the last SNR value indicates the absence of noise in the measurements. Peak-signal-to-noise ratio(PSNRs) is used to measure the quality of the restored images. In Table. 1 and Fig. 2 we report the reconstruction results we obtained using $T V$ and our proposed method $N S T V_{\delta}$. From these results we clearly observe that our proposed regularizer consistently better preforms than $T V$.

\subsection{Test on Image Debluring}

In this test, we test the performance of our proposed algorithm on image debluring problems whose objective is to reconstruct the unknown image $f^{*} \in \mathbb{R}^{N_{1} \times N_{2} \times M}\left(f^{*} \in\right.$ $\mathbb{R}^{N_{1} \times N_{2}}$ ) from an observed image $f=A f^{*}+n$ degraded by a linear blurring operator $A$ and a noise $n$, where we consider additive i.i.d. Gaussian noise $n$ with the noise level 


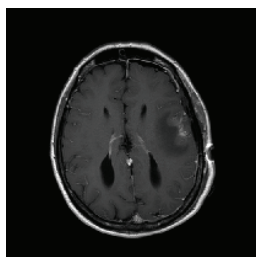

(a)

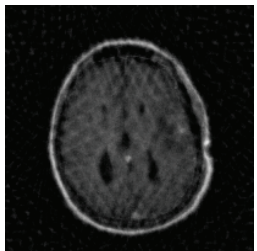

(b)

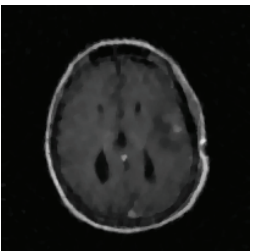

(c)

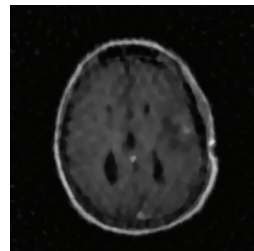

(d)

Figure 2: Reconstruct the image from Fourier data sampled with 48 radial lines and 20-dB SNR. (a) Original image; (b) Back-projiect image; (c) Our proposed nonconvex regularization method NSTV (d) $T V$ reconstruction.

$\sigma=0.0025$. Setting the stopping criterion are the same with stoping criterion in section 4.2. And the relative error defines as

$$
\text { RelErr }=\frac{\sum_{j=1}^{M}\left\|f_{j}-f_{j}^{*}\right\|_{F}^{2}}{\sum_{j=1}^{M}\left\|f_{j}^{*}\right\|_{F}^{2}} .
$$

To simplify the presentation, we also use $\left\|f-f^{*}\right\|^{2} /\left\|f^{*}\right\|^{2}$ to denote relative error. In Fig. 3, for the vector-value image test, we show the experimental results with the wellknown "Lena" image which contains avariety of meaningful parts in it, such as the large flat area, the areas with gradual changes of the image intensity and the areas with different shapes. During this experiment, the first image is corrupted by "Gaussian" blur $(\mathrm{PSNR}=25.558)$ with standard deviation $\sigma=1.5$ pixels, truncated in a $5 \times 5$ pixel window. The second image "House" blurred by "motion" kernel (PSNR=22.152) with standard deviation $\sigma=1.5$ pixels, truncated in a $15 \times 15$ pixel window.

In Fig. 4, we give the relative error values of these methods, and in table 2, we give the PSNRs of these methods. In Fig. 5, for grayscale image deblur test, we show the experimental results with the well-known "Cameraman" and "Lenna". During this experiment, the first image is corrupted by "Gaussian" blur (PSNR=22.593) with standard deviation $\sigma=1.5$ pixels, truncated in a $5 \times 5$ pixel window. The second image blurred by "motion" kernel (PSNR=18.481) with standard deviation $\sigma=1.5$ pixels, truncated in a $15 \times 15$ pixel window. 

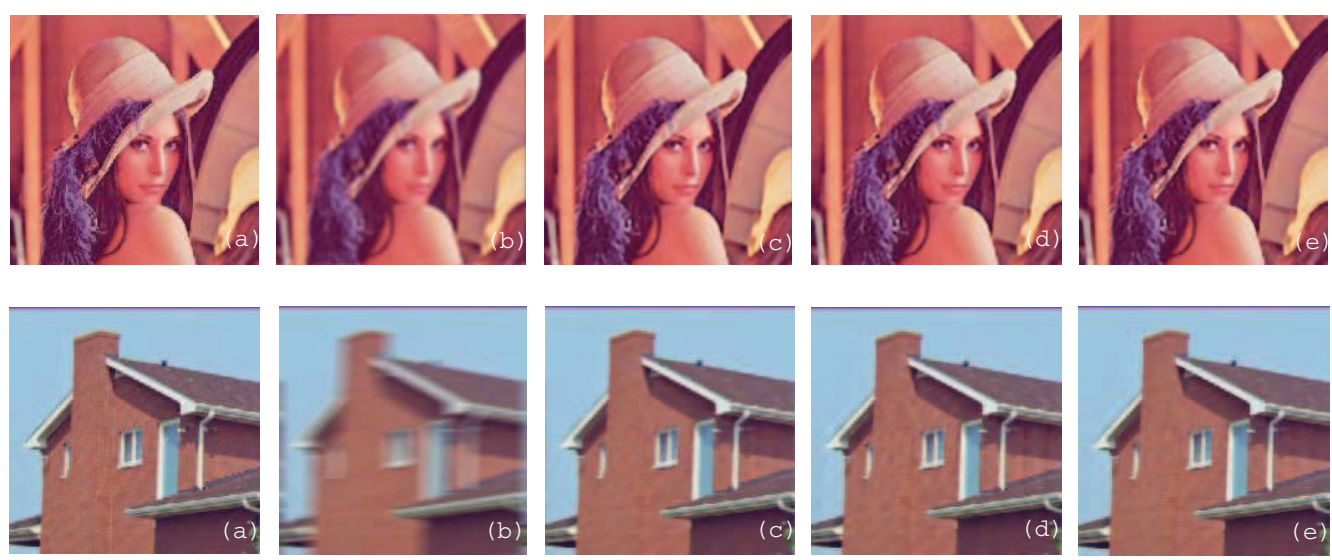

Figure 3: (a) Original vector-value images; (b) Blurred images; (c) Recovered images by $T V$; (d) Recovered images by $S T V_{\delta} ;$ (e) Recovered images by $N S T V_{\delta}$.

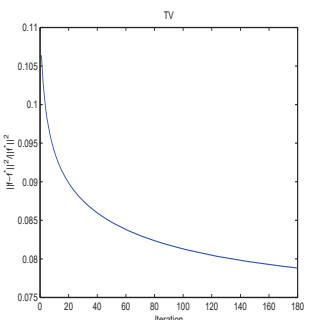

(a)

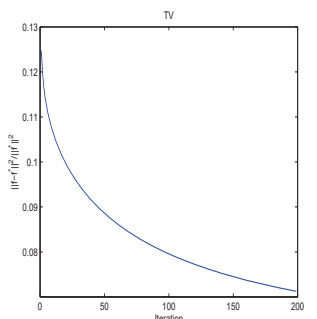

(d)

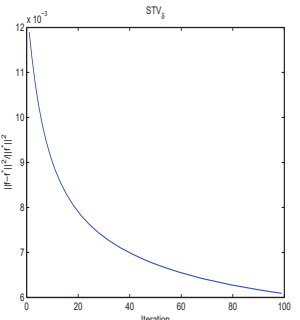

(b)

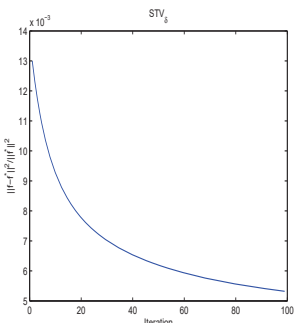

(e)

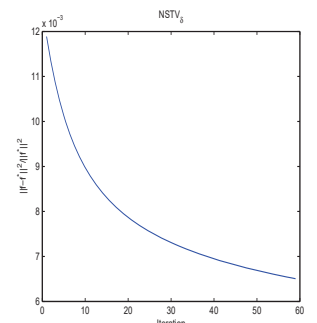

(c)

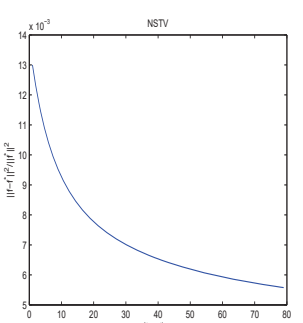

(f)

Figure 4: Relative error values of (a) recovered image (Lenna) by $T V$; (b) recovered image (Lenna) by $S T V_{\delta}$; (c) recovered image (Lenna) by $N S T V_{\delta}$; (d) recovered image (House) by $T V$; (e) recovered image (House) by $S T V_{\delta} ;$ (f) recovered image (House) by $N S T V_{\delta}$. 

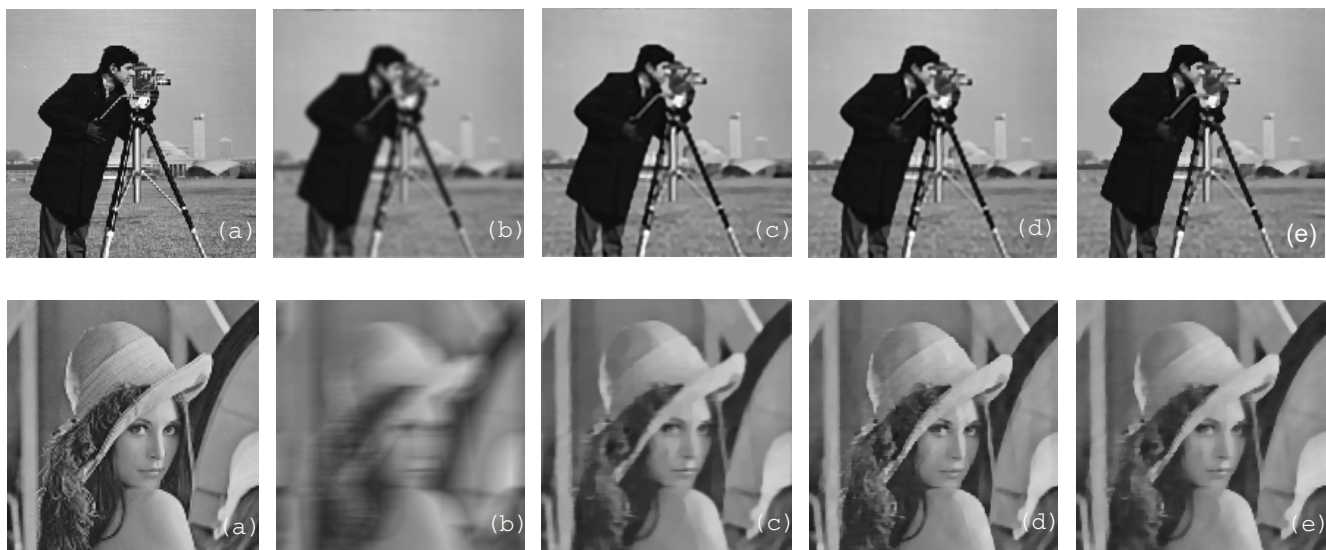

Figure 5: (a) Original grayscale images; (b) Blurred images [psnr=22.593 (first image), 18.481 (second image)]; (c) Recovered images by $T V$ [psnr=26.691 (first image), 27.197 (second image)]; (d) Recovered images by $S T V_{\delta}$ [psnr=27.153 (first image), 27.208 (second image)]; (e) Recovered images by $N S T V_{\delta}$ [psnr=27.617 (first image), 27.345 (second image)].

\subsection{Test on Image Denoise}

In this test, we test the performance of our proposed algorithm on image denoising problems whose objective is to reconstruct the unknown image $f^{*} \in \mathbb{R}^{N_{1} \times N_{2} \times M}(\hat{f} \in$ $\mathbb{R}^{N_{1} \times N_{2}}$ ) from an observed image $f=f^{*}+n$, where we consider additive i.i.d. Gaussian noise $n$ with the noise level $\sigma=0.1$. Regarding the stopping criterion for the optimization algorithm are the same with stoping criterion in section 3.2. In Fig. 6, for the vectorvalue image test, we show the experimental results with the well-known "Flower" image. During this experiment, the image is corrupted by "Gaussian" noise $(\mathrm{PSNR}=23.027)$ with standard deviation $\sigma=0.1$, (f) and (g) images are recovered by $S T V_{K}$ and our proposed $N S T V_{K}$ regularization methods, where $K$ is the convolution kernel, we choose it to be a Gaussian with standard deviation $\sigma=0.5$ pixels, truncated in a $5 \times 5$ pixel window.

For the grayscale image test, we show the experimental results with the well-known "Baboon" image which contains great texture and structure information. The noise added in it is also the white Gaussian noise with standard variance $\sigma=0.1(\mathrm{PSNR}=21.491)$ and the restored images by these five approaches are shown in Fig. 8. (f) and (g) images 205 are recovered by $S T V_{K}$ and our proposed $N S T V_{K}$ regularization methods, where $K$ is 


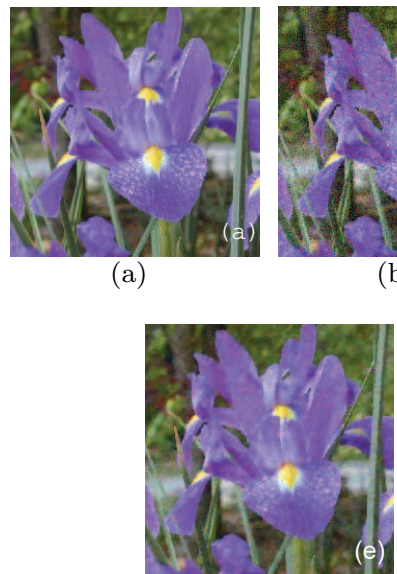

(e) (b)

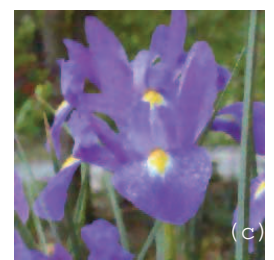

(c)

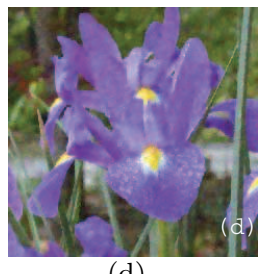

(d)

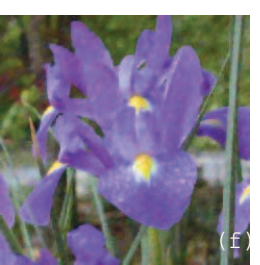

(f)

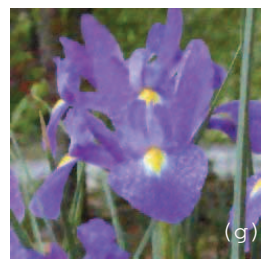

(g)

Figure 6: (a) Original vector-value image; (b) Noising image; (c) Recovered image by $T V$; (d) Recovered image by $S T V_{\delta}$; (e) Recovered image by $N S T V_{\delta}$ (f) Recovered image by $S T V_{K}$; (g) Recovered image by $N S T V_{K}$.

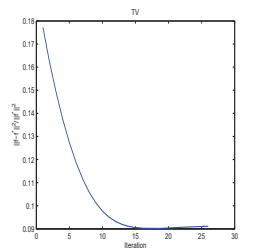

(a)

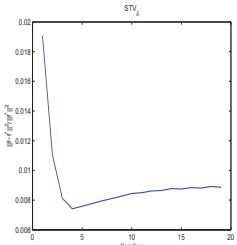

(b)

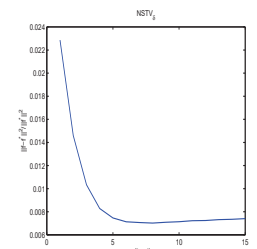

(c)

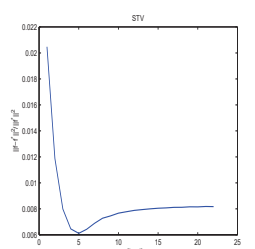

(d)

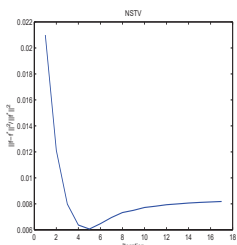

(e)

Figure 7: Relative error values of (a) recovered image by $T V$; (b) recovered image by $S T V_{\delta}$; (c) recovered image by $N S T V_{\delta} ;$ (d) recovered image by $S T V_{K}$; (e) recovered image by $N S T V_{K}$. 


\begin{tabular}{c|rrr|rrr|rrr}
\hline \hline \multirow{2}{*}{ Methods } & \multicolumn{3}{|c|}{ Lenna } & \multicolumn{3}{|c}{ House } & \multicolumn{3}{|c}{ Flower } \\
& PSNR(dB) & $\sharp I$ Iter & Time $(\mathrm{s})$ & PSNR(dB) & $\sharp$ Iter & Time(s) & PSNR(dB) & $\sharp I t e r$ & Time(s) \\
\hline$T V$ & 27.419 & 180 & $\mathbf{1 6 . 3 2}$ & 27.254 & 191 & $\mathbf{2 8 . 6 6}$ & 27.317 & 26 & $\mathbf{3 . 1 7}$ \\
$S T V_{\delta}$ & 27.496 & 99 & 265.28 & 27.348 & 97 & 197.59 & 27.488 & 19 & 54.61 \\
$N S T V_{\delta}$ & $\mathbf{2 7 . 5 9 2}$ & $\mathbf{5 9}$ & 212.62 & $\mathbf{2 7 . 4 4 5}$ & $\mathbf{7 9}$ & 160.41 & $\mathbf{2 7 . 8 5 4}$ & $\mathbf{1 5}$ & 70.91 \\
$S T V_{K}$ & -- & -- & -- & -- & -- & -- & 27.442 & 22 & 109.63 \\
$N S T V_{K}$ & -- & -- & -- & -- & -- & -- & 27.501 & 17 & 108.37 \\
\hline \hline
\end{tabular}

the convolution kernel, we choose it to be a Gaussian with standard deviation $\sigma=0.5$ pixels, truncated in a $5 \times 5$ pixel window.

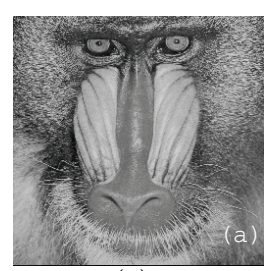

(a)

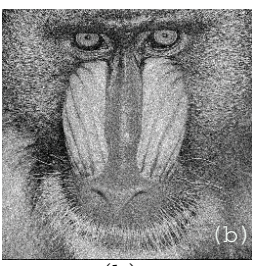

(b)

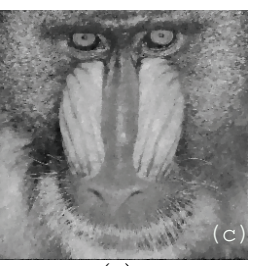

(c)

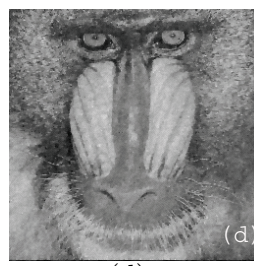

(d)

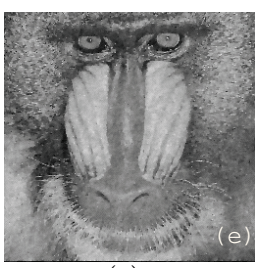

(e)

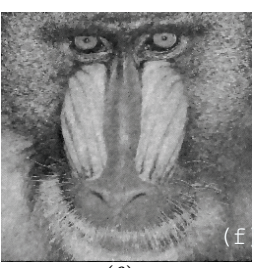

(f)

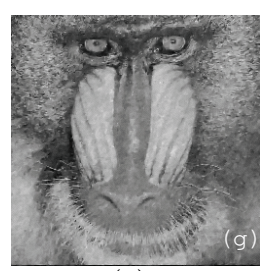

(g)

Figure 8: (a) Original grayscale image; (b) Noising image (psnr=21.491); (c) Recovered image by $T V$ (psnr=21.536); (d) Recovered image by $S T V_{\delta} \quad\left(\right.$ psnr=21.543); (e) Recovered image by $N S T V_{\delta}$ (psnr=21.590); (f) Recovered image by $S T V_{K}(\mathrm{psnr}=21.564) ;(\mathrm{g})$ Recovered image by $N S T V_{K}$ (p$\operatorname{snr}=21.574)$.

As we can see in the above experiment results, the performance (relative error and PSNR) of our proposed methods are better than other methods in this paper. 


\section{5. conclusions}

In this paper, we have proposed a novel regularization method for image reconstruction and restoration, which is accomplished by adopting a nonconvex nonsmooth penalty that depends on the eigenvalues of structure tensor of the underlying image. And our theoretical result indicates that there exist a sequence which is generated by our algorith$\mathrm{m}$ converges to a critical point. The efficiency of the method are performed via various numerical simulations. In addition, we plan to construct a high order structure tensor in the future work, which can generalize the mean curvature or total generalized variation in grayscale images and vector-value images.

\section{Acknowledgments}

We thank the anonymous referees for their detailed comments, which helped to improve the paper.

\section{Appendix}

(A) Proof of Proposition 2.1. We have $\tilde{\Psi}(u)=\sum_{i=1}^{N_{1}} \sum_{k=1}^{N_{2}}\left[\psi\left(\sqrt{\lambda_{i, k}^{+}}\right)+\psi\left(\sqrt{\lambda_{i, k}^{-}}\right)\right]$, $\tilde{\Psi}: \mathbb{R}^{N_{1} \times N_{2} \times M} \rightarrow \mathbb{R}$. From $[20]$ we know that $\psi \in \mathcal{C}^{2}(\mathbb{R})$, then $D_{u} \tilde{\Psi}(u) \in L\left(\mathbb{R}^{N_{1} \times N_{2} \times M}, \mathbb{R}\right)$, and

$$
\left(D_{u} \tilde{\Psi}(u)\right)_{i, k}=\frac{\partial \psi}{\partial \lambda_{i, k}^{+}} \cdot D_{u} \lambda_{i, k}^{+}(u)+\frac{\partial \psi}{\partial \lambda_{i, k}^{-}} \cdot D_{u} \lambda_{i, k}^{-}(u),
$$

where $i=1, \ldots, N_{1}$ and $k=1, \ldots, N_{2}$. On the other hand, we have

$$
D_{u} \lambda_{i, k}^{+}=\left\langle D_{\left(S_{K} u\right)_{i, k}} \lambda_{i, k}^{+}, D_{u}\left(S_{K} u\right)_{i, k}\right\rangle
$$

And another form of $\left(S_{K} u\right)_{i, k}$ is

$$
\left(S_{K} u\right)_{i, k}=\lambda_{i, k}^{+} \theta_{i, k}^{+}\left(\theta_{i, k}^{+}\right)^{T}+\lambda_{i, k}^{-} \theta_{i, k}^{-}\left(\theta_{i, k}^{-}\right)^{T}
$$

Multiplying (5.1) by $\left(\theta_{i, k}^{+}\right)^{T}$ on the left and by $\theta_{i, k}^{+}$on the right, we obtain

$$
\lambda_{i, k}^{+}=\left\langle\theta_{i, k}^{+}\left(\theta_{i, k}^{+}\right)^{T},\left(S_{K} u\right)_{i, k}\right\rangle
$$


where $\left\langle\theta_{i, k}^{+}\left(\theta_{i, k}^{+}\right)^{T},\left(S_{K} u\right)_{i, k}\right\rangle=\operatorname{Tr}\left(\theta_{i, k}^{+}\left(\theta_{i, k}^{+}\right)^{T}\left(S_{K} u\right)_{i, k}\right)$, so we have

$$
D_{\left(S_{K} u\right)_{i, k}} \lambda_{i, k}^{+}=\theta_{i, k}^{+}\left(\theta_{i, k}^{+}\right)^{T} .
$$

What's more, we know that $\left(S_{K} u\right)_{i, k}=\left(J_{K} u\right)_{i, k}^{T}\left(J_{K} u\right)_{i, k},\left(J_{K} u\right)_{i, k}=\left(J_{K} u\right)(i, k,:,:)$, and we set the operator $P_{i, k}: \mathbb{R}^{N_{1} \times N_{2} \times L_{K} \cdot M \times 2} \rightarrow \mathbb{R}^{L_{K} \cdot M \times 2}$ to be $P_{i, k} J_{K} u=\left(J_{K} u\right)_{i, k}$. Thus, we have

$$
D_{u}\left(S_{K} u\right)_{i, k}=\left(P_{i, k} J_{K} u\right)^{T} P_{i, k} J_{K}+\left(P_{i, k} J_{K}\right)^{T} P_{i, k} J_{K} u .
$$

From above analysis, we know that

$$
D_{u} \lambda_{i, k}^{+}=2 J_{K}^{*} P_{i, k}^{*}\left(\theta_{i, k}^{+}\left(\theta_{i, k}^{+}\right)^{T} P_{i, k} J_{K} u\right),
$$

where $P_{i, k}^{*}$ is the adual of $P_{i, k}$, and we have

$$
\left(D_{u} \tilde{\Psi}(u)\right)_{i, k}=2 J_{K}^{*} P_{i, k}^{*}\left(\Theta_{i, k} P_{i, k} J_{K} u\right),
$$

$225 \quad$ where $\Theta_{i, k}=\frac{\partial \psi\left(\sqrt{\lambda_{i, k}^{+}}\right)}{\partial \lambda_{i, k}^{+}} \cdot \theta_{i, k}^{+}\left(\theta_{i, k}^{+}\right)^{T}+\frac{\partial \psi\left(\sqrt{\lambda_{i, k}^{-}}\right)}{\partial \lambda_{i, k}^{-}} \cdot \theta_{i, k}^{-}\left(\theta_{i, k}^{-}\right)^{T}$.

(B) Proof of Proposition 3.1. The inequalities (3.1) and (3.3) can be obtained by direct computation. The proof for the inequality of (3.2) can be considered as follows.

$$
\begin{aligned}
& L_{\rho}\left(u^{(s)}, W^{(s)}, P^{(s-1)}\right) \leq L_{\rho}\left(u^{(s-1)}, W^{(s)}, Z^{(s-1)}\right) \\
& \quad+\left\langle D_{u} L_{\rho}\left(u^{(s-1)}, W^{(s)}, Z^{(s-1)}\right), u^{(s)}-u^{(s-1)}\right\rangle+\frac{L}{2}\left\|u^{(s)}-u^{(s-1)}\right\|_{F, 1}^{2},
\end{aligned}
$$

where $L$ is a Lipschitz constant of $D_{X} L_{\rho}\left(X^{(s)}, W^{(s+1)}, Z^{(s)}\right)$. By (2.5), we have

$$
\begin{aligned}
& L_{\rho}\left(u^{(s)}, W^{(s)}, P^{(s-1)}\right) \leq L_{\rho}\left(u^{(s-1)}, W^{(s)}, P^{(s-1)}\right) \\
& \quad-\left\langle\left(A^{*} A+\rho J_{K}^{*} J_{K}\right)\left(u^{(s)}-u^{(s-1)}\right), u^{(s)}-u^{(s-1)}\right\rangle+\frac{L}{2}\left\|u^{(s)}-u^{(s-1)}\right\|_{F, 1}^{2} .
\end{aligned}
$$

We have

$$
L_{\rho}\left(u^{(s)}, W^{(s)}, P^{(s-1)}\right)+\left(\underline{\lambda}-\frac{L}{2}\right)\left\|u^{(s)}-u^{(s-1)}\right\|_{F, 1}^{2} \leq L_{\rho}\left(u^{(s-1)}, W^{(s)}, Z^{(s-1)}\right) .
$$

From the assumption of the proposition, i.e, $\underline{\lambda}-\frac{L}{2}>0$, the inequality (3.2) hold.

(c) Proof of Theorem 3.1. According to (2.6) and (2.7), we have

$$
\begin{aligned}
0 & \in \partial_{W} L_{\rho}\left(u^{(s-1)}, W^{(s)}, P^{(s-1)}\right) \\
& =\tau \alpha \partial\left\|W^{(s)}\right\|_{*, 1}+P^{(s-1)}-\rho\left(W^{(s)}-J_{K} u^{(s-1)}\right),
\end{aligned}
$$


and

$$
\begin{aligned}
- & \left(A^{*} A+\rho J_{K}^{*} J_{K}\right)\left(u^{(s)}-u^{(s-1)}\right)=D L_{\rho}\left(u^{(s-1)}, W^{(s)}, P^{(s-1)}\right) \\
& =A^{*}\left(A u^{(s-1)}-g\right)+\tau D \Psi\left(u^{(s-1)}\right)-J_{K}^{*} P^{(s-1)}+\rho J_{K}^{*}\left(W^{(s)}-J_{K} u^{(s-1)}\right) .
\end{aligned}
$$

From the definition of the augmented Lagrangian function (2.5), we have

$$
\begin{gathered}
\partial_{W} L_{\rho}\left(X^{(s)}, W^{(s)}, Z^{(s)}\right) \\
=\lambda \alpha \partial\left\|W^{(s)}\right\|_{*, 1}+P^{(s)}-\rho\left(W^{(s)}-J_{K} u^{(s)}\right), \\
D_{u} L_{\rho}\left(u^{(s)}, W^{(s)}, Z^{(s)}\right) \\
=A^{*}\left(A u^{(s)}-g\right)+\tau D \Psi\left(u^{(s)}\right)-J_{K}^{*} P^{(s)}+\rho J_{K}^{*}\left(W^{(s)}-J_{K} u^{(s)}\right),
\end{gathered}
$$

With (5.2) and (5.4), we obtain

$$
\partial_{W} L_{\rho}\left(u^{(s)}, W^{(s)}, P^{(s)}\right)=P^{(s)}-P^{(s-1)}+\rho\left(J_{K} u^{(s)}-J_{K} u^{(s-1)}\right) .
$$

With (5.3) and (5.5), we obtain

$$
D_{u} L_{\rho}\left(u^{(s)}, W^{(s)}, P^{(s)}\right)=\tau D \Psi\left(u^{(s)}\right)-\tau D \Psi\left(u^{(s-1)}\right)+J_{K}^{*} P^{(s-1)}-J_{K}^{*} P^{(s)} .
$$

And via (2.5), we have

$$
D_{P} L_{\rho}\left(u^{(s)}, W^{(s)}, P^{(s)}\right)=\frac{1}{\rho}\left(P^{(s)}-P^{(s-1)}\right) .
$$

From [1], we known that there exists a $\kappa>0$, and

$$
\kappa \cdot \operatorname{dist}\left(\partial L_{\rho}\left(u^{(s)}, W^{(s)}, P^{(s)}\right), 0\right)^{2} \leq L_{\rho}\left(u^{(s-1)}, W^{(s-1)}, P^{(s-1)}\right)-L_{\rho}\left(u^{(s)}, W^{(s)}, P^{(s)}\right) .
$$

Then, (3.4) hold.

(D) Proof of Theorem 3.2. From (3.1), (3.2) and (3.3), we know that the sufficient decrease condition [1] is satisfied. Therefore we only need to prove

(1) relative error condition, i.e., there exists $\left(\widehat{u}^{(s)}, \widehat{W}^{(s)}, \widehat{P}^{(s)}\right) \in \partial L_{\rho}\left(u^{(s)}, W^{(s)}, P^{(s)}\right)$ 235 such that

$$
\begin{aligned}
\left\|\widehat{u}^{(s)}\right\|_{F, 1} & +\left\|\widehat{W}^{(s)}\right\|_{F, 1}+\left\|\widehat{P}^{(s)}\right\|_{F, 1} \\
& \leq b\left(\left\|u^{(s)}-u^{(s-1)}\right\|_{F, 1}+\left\|W^{(s)}-W^{(s-1)}\right\|_{F, 1}+\left\|P^{(s)}-P^{(s-1)}\right\|_{F, 1}\right) .
\end{aligned}
$$


(2) continuity condition, i.e., there exists a subsequence $\left(u^{\left(s_{j}\right)}, W^{\left(s_{j}\right)}, P^{\left(s_{j}\right)}\right)$ such that

$$
\begin{aligned}
& \left(u^{(s)}, W^{(s)}, P^{(s)}\right) \rightarrow\left(u^{*}, W^{*}, P^{*}\right), \\
& L_{\rho}\left(u^{(s)}, W^{(s)}, P^{(s)}\right) \rightarrow L_{\rho}\left(u^{*}, W^{*}, P^{*}\right) \text {, as } j \rightarrow+\infty .
\end{aligned}
$$

First, Define

$$
\left\{\begin{array}{l}
\widehat{u}^{(s)}=P^{(s)}-P^{(s-1)}+\rho\left(J_{K} u^{(s)}-J_{K} u^{(s-1)}\right) \\
\widehat{W}^{(s)}=\tau D \Psi\left(u^{(s)}\right)-\tau D \Psi\left(u^{(s-1)}\right)+J_{K}^{*} P^{(s-1)}-J_{K}^{*} P^{(s)} \\
\widehat{P}^{(s)}=\frac{1}{\rho}\left(P^{(s)}-P^{(s-1)}\right) .
\end{array}\right.
$$

Let $b=\tau L^{\prime}+\rho+\frac{1}{\rho}+1+(1+\rho) \bar{\lambda}$ and $\bar{\lambda}=\lambda_{\max } \sqrt{\left(J_{K}^{*} J_{K}\right)}$, then, (5.6) holds, where $D \hat{\Psi}$ is $L^{\prime}$-Lipschitz function. By the semi-continuity of $L_{\rho}(u, W, P)$, it is easy to know that (5.7) also holds.

\section{References}

[1] H. Attouch, J. Bolte, P. Redont, A. Soubeyran, Proximal alternating minimization and projection methods for nonconvex problems: an approach based on the Kurdyka-Łojasiewicz inequality, Math. Oper. Res. 35 (2010) 438-457.

[2] P. Blomgren, T. F. Chan, Color TV: total variation methods for restoration of vector-valued images, IEEE Trans. Image Process. 7 (1998) 304-309.

[3] J.-F. Cai, E. J. Candès, Z. Shen, A singular value thresholding algorithm for matrix completion, SIAM J. Optim. 20 (2010) 1956-1982.

[4] E. J. Candès, J. Romberg, T. Tao, Robust uncertainty principles: Exact signal reconstruction from highly incomplete frequency information, IEEE Trans. Inform. Theory 52 (2006) 489-509.

[5] A. Chambolle, Image recovery via total variation minimization and related problems, Numerische Mathematik 76 (1997) 167-188.

[6] A. Chambolle, An algorithm for total variation minimization and applications, J. Math. Imaging Vis. 20 (2004) 89-97.

[7] Z.-X. Cui, Q. Fan, A nonconvex nonsmooth regularization method for compressed sensing and low-rank matrix completion, arXiv preprint arXiv: 1605.00479.

[8] G. Demoment, Image reconstruction and restoration: overview of common estimation structures and problems, IEEE Trans. Acoust. Speech Signal Process. 37 (1989) 2024-2036.

[9] V. Estellers, S. Soatto, X. Bresson, Adaptive regularization with the structure tensor, IEEE Trans. Image Process. 24 (2015) 1777-1790.

260 [10] J. Fan, R. Li, Variable selection via nonconcave penalized likelihood and its oracle properties, J. Am. Stat. Assoc. 96 (2011) 1348-1360. 
[11] W. Förstner, E. Gúlch, A fast operator for detection and precise location of distinct points, corners and circular features, Proc. ISPRS Intercommission Conf. Fast Process. of Photogrammetric Data 96 (1987) 281-305.

5 [12] L. E. Frank, J. H. Friedman, A statistical view of some chemometrics regression tools, Technometrics 35 (1993) 109-135.

[13] S. Ghadimi, G. Lan, Stochastic first- and zeroth-order methods for nonconvex stochastic programming, SIAM J. Optim. 23 (2013) 2341-2368.

[14] C. Hoelen, F. F. M. De Mul, R. Pongers, A. Dekker, Three-dimensional photoacoustic imaging of blood vessels in tissue, Optics Letters 23 (1998) 648-650.

[15] Y. Hu, D. Zhang, J. Ye, X. Li, X. He, Fast and accurate matrix completion via truncated nuclear norm regularization, IEEE Trans. Pattern Anal. Mach. Intell. 35 (2013) 2117-2130.

[16] K. Ito, B. Jin, Models in inverse problems, in: Inverse Problems: Tikhonov Theory and Algorithms, World Scientific Press (2015) 5-25.

275 [17] Z. Jin, Z. Wan, Y. Jiao, X. Lu, An alternating direction method with continuation for nonconvex low rank minimization, J. Sci. Comput. 66 (2015) 849-869.

[18] S. Lefkimmiatis, A. Roussos, P. Maragos, M. Unser, Structure tensor total variation, SIAM J. Imag. Sci. 8 (2015) 1090-1122.

[19] S. Lefkimmiatis, M. Unser, Hessian Schatten-norm regularization for linear inverse problems, IEEE Trans. Image Process. 22 (2013) 1873-1888.

[20] M. Nikolova, M. K. Ng, C. Tam, Fast nonconvex nonsmooth minimization methods for image restoration and reconstruction, IEEE Trans. Image Process. 19 (2010) 3073-3088.

[21] M. Nikolova, M. K. Ng, C. Tam, On $\ell_{1}$ data fitting and concave regularization for image recovery, SIAM J. Sci. Comput. 35 (2013) A397-A430.

[22] L. I. Rudin, S. Osher, E. Fatemi, Nonlinear total variation based noise removal algorithms, Physica D: Nonlinear Phenomena 60 (1992) 259-268.

[23] O. Scherzer, M. Grasmair, H. Grossauer, M. Haltmeier, F. Lenzen, Image and noise models, in: Variational Methods in Imaging, Applied Mathematical Sciences, Springer (2009) 27-46.

[24] D. L. Snyder, A. M. Hammoud, Image recovery from data acquired with a charge-coupled-device camera, Journal of The Optical Society of America A-optics Image Science and Vision 10 (1993) 1014-1023.

[25] D. Tao, J. Cheng, M. Song, X. Lin, Manifold ranking-based matrix factorization for saliency detection, IEEE Trans. Neural Networks 27 (2016) 1122-1134.

[26] D. Tao, Y. Guo, M. Song, Y. Li, Z. Yu, Y. Y. Tang, Person re-identification by dual-regularized kiss metric learning, IEEE Trans. Image Process. 25 (2016) 2726-2738.

[27] D. Tao, L. Jin, W. Liu, X. Li, Hessian regularized support vector machines for mobile image annotation on the cloud, IEEE Trans. Multimedia 15 (2013) 833-844.

[28] D. Tschumperle, R. Deriche, Diffusion PDEs on vector-valued images, IEEE Signal Process. Mag. 19 (2002) $16-25$.

300 [29] D. Tschumperle, R. Deriche, Vector-valued image regularization with PDE's: a common framework 
for different applications, IEEE Trans. Pattern Anal. Mach. Intell. 27 (2005) 506-517.

[30] C. R. Vogel, Computational Methods for Inverse Problems, Frontiers in Applied Mathematics, SIAM, 2002.

[31] J. Xiao, M. K. Ng, Y. Yang, On the convergence of nonconvex minimization methods for image recovery, IEEE Trans. Image Process. 24 (2015) 1587-1598.

[32] J. Yu, Y. Rui, Y. Y. Tang, D. Tao, High-order distance-based multiview stochastic learning in image classification, IEEE Trans. Systems, Man, and Cybernetics 44 (2014) 2431-2442.

[33] J. Yu, D. Tao, M. Wang, Y. Rui, Learning to rank using user clicks and visual features for image retrieval, IEEE Trans. Systems, Man, and Cybernetics 45 (2015) 767-779.

[34] J. Yu, X. Yang, F. Gao, D. Tao, Deep multimodal distance metric learning using click constraints for image ranking, IEEE Trans. Systems, Man, and Cybernetics.

[35] S. D. Zenzo, A note on the gradient of a multi-image, Computer Vision, Graphics and Image Process. 33 (1986) 116-125.

[36] C. Zhang, Nearly unbiased variable selection under minimax concave penalty, Annals of Statistics 38 (2010) 894-942. 